\title{
Evaluation of the Dairy Comp 305 Module "Cow Value" in Two Ontario Dairy Herds
}

\author{
U. S. Sorge,${ }^{\star 1}$ D. F. Kelton, ${ }^{*}$ K. D. Lissemore, ${ }^{*}$ W. Sears,${ }^{*}$ and J. Fetrow† \\ *Department of Population Medicine, University of Guelph, Guelph, Ontario, Canada \\ †Department of Veterinary Population Medicine, University of Minnesota, St. Paul 55108
}

\begin{abstract}
The study was conducted to evaluate how the "Cow Value" module of Dairy Comp 305 (Valley Agricultural Software, Tulare, CA) performed under commercial conditions. The "Cow Value" module, COWVAL, computes a farm-specific net present value relative to an average replacement heifer for each cow in the milking and dry herd, which allows a ranking of the cows on the farm compared with replacing her with a typical replacement heifer on that farm. The average replacement heifer is used as the baseline for comparison and has a COWVAL of $\$ 0$. Retaining a cow with a negative COWVAL is projected to be less profitable than replacing that cow with a new heifer. The objectives of the study were to explore trends in COWVAL over and during multiple lactations for the same cows; to describe factors that influence changes in COWVAL from one monthly Dairy Herd Improvement test to the next; and to evaluate the behavior of COWVAL after it drops below a baseline of $\$ 0$ during the lifetime of a cow. Monthly Dairy Comp 305 backup cow files from 2 Ontario dairy herds between December 1999 and December 2005 were used to generate COWVAL and list production, reproduction, and disease data for the milking cows. In total, 1,463 cows and 20,071 tests were analyzed. Within the first $60 \mathrm{~d}$ in milk (DIM), COWVAL was unstable and showed large fluctuations over a range of several thousand Canadian dollars (Can\$). After 60 DIM COWVAL was relatively stable. The variability from month to month became less as the lactation progressed and the risk of a change in reproductive status decreased. The reproductive status of the cow influenced COWVAL: fresh, open, and pregnant cows had a greater COWVAL than cows declared "do not breed." As parity increased, there was a tendency toward lower COWVAL and smaller monthly changes in COWVAL.
\end{abstract}

Received December 4, 2006.

Accepted August 10, 2007.

${ }^{1}$ Corresponding author: usorge@uoguelph.ca
The COWVAL of 170 cows dropped below the baseline of $\$ 0$ after 60 DIM. The COWVAL of $54 \%$ of those cows remained below $\$ 0$, whereas $31.6 \%$ had a subsequent COWVAL $>\$ 500(\mathrm{Can} \$)$. Farm management should not rely exclusively on COWVAL for culling decisions, particularly for cows that have not had at least 3 milk tests. Key words: culling, economics, dairy cow

\section{INTRODUCTION}

All dairy cows are eventually culled or replaced. Typically, cows stay in the milking herd for $<4 \mathrm{yr}$ before they are culled (Lehenbauer, 1998). The terms culling, replacement, and removal are considered synonymous and will be used interchangeably in this article.

About 25 to $35 \%$ of milking cows in a dairy herd will be replaced each year (Lehenbauer, 1998). The reasons for exit vary, but they can be divided into the 2 broad categories of forced and economic culls (Fetrow et al., 2006). The latter represents the active decision of the farmer, who projects a better return if the existing cow is replaced by a new heifer. Because milk represents up to $90 \%$ of the income of a dairy farm, the production level of a cow, both current and projected, is the primary basis for the culling decision. A high milk yield, relative to herd mates, is protection against culling (Dijkhuizen et al., 1984/85; Beaudeau et al., 1995; Gröhn et al., 1998).

The factors commonly considered in economic culling decisions are milk production, reproductive status, age, health status, stage of lactation, the cost and availability of replacement animals, calving patterns, genetic improvement goals, and the herd production relative to quota holdings in Europe and Canada (Beaudeau et al., 1996; Radke and Lloyd, 2000; Gröhn et al., 2003). As part of the culling decision, the farmer makes assumptions about the present and future performance and profitability of a cow compared with a possible replacement (usually a heifer). Dairy managers usually rely on their experience and intuition to make this decision (Lehenbauer and Oltjen, 1998).

Over the years, various models have been developed to rank the cows in a herd to help producers with their 
replacement decisions (Kristensen, 1987; Groenendaal et al., 2004). Unfortunately these models have not become broadly accessible or operational. In the fall of 1999, the dairy herd management computer program Dairy Comp 305 (DC 305; Valley Agricultural Software, Tulare, CA) introduced a new functional module called "Cow Value." Cow Value is conceptually similar to the previously mentioned models; it computes a farm-specific value for the future profit to the dairy if the existing cow is retained vs. being replaced by an average replacement heifer for the dairy. The calculation computes the net present value (NPV) of projected income minus feed and replacement costs for the cow in the relevant future (i.e., until the demographics of the herd are the same whether the cow is culled today or not and until discounting renders any future financial impacts irrelevant). Cows retained in the herd (i.e., not culled) are assumed to continue their lactation, continue to be bred if not pregnant, and to suffer attrition in their future the same as other retained cows. Having calculated the NPV projection for retaining the cow, the same calculation is done for a potential average replacement heifer that could replace the existing cow today. The difference between those 2 NPV calculations is the Cow Value (COWVAL). Thus, COWVAL makes it possible to rank the cows on the farm compared with the potential of a typical replacement heifer on that farm. The average replacement heifer, the baseline for comparison, has a COWVAL of $\$ 0$. Hence, cows that have a positive COWVAL are economically more valuable to that farm than an average replacement heifer and should stay in the herd. In contrast, cows that have a negative COWVAL are less profitable and should be considered for replacement. In addition, COWVAL may be used to contribute to other management decisions on the dairy; for example, decisions to breed a cow or when considering treatment of sick cows (Eicker and Fetrow, 2003). For example, a cow with a displaced abomasum and a COWVAL of $\$ 100$ might be more profitably culled than treated.

The factors included in the estimation of COWVAL are farm and cow specific. At the farm level the program uses farm-specific inputs for costs of feed and replacement heifers, cull price, milk price, discount rate, detection of estrus and conception rates, voluntary waiting period, average days open, culling risk for the various lactations, and the annual milk production and milk curve persistency of lactations 1,2 , and $\geq 3$. At the individual cow level, the age of the cow, stage of lactation, reproductive status, and production level are taken into consideration. Breeding costs, temperament, and genetic pedigree are not considered in the calculation of COWVAL. Disease history and current health status are not considered, except indirectly through the effects reflected in milk production (Eicker and Fetrow, 2003).

Although DC 305 is widely used on dairies in Canada and the United States, the number of farmers using COWVAL as part of their decision-making process is not known. No formal evaluation of how COWVAL behaves under field conditions has been reported to date. This study was conducted to explore trends in monthly calculations of COWVAL over and during various lactations for a sample of cows. The study sought to describe factors that influenced changes in the COWVAL from one monthly DHI test date to the next and to evaluate the behavior of COWVAL after an individual COWVAL dropped below the baseline of $\$ 0$.

\section{MATERIALS AND METHODS}

A convenience sample of 2 dairy herds in southwestern Ontario was selected. The herds were chosen because the farm managers used DC 305 as their onfarm herd management software, the herds had been enrolled in Ontario/CanWest DHI milk recording since 1999, and all reproductive event data were recorded routinely. Monthly DC 305 backup cow files from December 1999 to December 2005 for the 2 farms were used to generate COWVAL retrospectively for all cows in the herd following each DHI test day. The default herd values used as input variables for COWVAL were fixed throughout the study to avoid changes in COWVAL due to changes in these herd-level factors. For both farms, the cost of raising a heifer, cull price, feed costs (marginal and maintenance), discount rate, and culling probabilities for cows in lactation $\geq 6$ were adjusted to meet the current Canadian industry standards (Table 1; Lang, 2003; Gencor, 2006). All dollar values reported herein are in Canadian dollars (Can\$). The price of $100 \mathrm{~kg}$ of milk was set at $\$ 40$, considerably less than the market price, to account for the carrying costs of quota in Canada. Farm-specific mean estrous detection rate, conception rate, and milk yield for lactations 1 through 3 were determined by averaging their values for the months of January, March, May, July, and October of each year of the study period (Table 1).

To create the data set, the monthly DC 305 backup cow files were used to generate and extract the COWVAL for each cow, each month, based on the fixed farmspecific default values, and to list various production, reproduction, and disease variables for each cow in the herd. These monthly herd-specific observations of the cows in the 2 herds were subsequently combined into a single data set.

The changes in COWVAL were calculated from one DHI test date to the next (approximately month to month) and will subsequently be called DELTA. The 
Table 1. Default values applied for the computation of COWVAL ${ }^{1}$ for the cows in the 2 Ontario dairy herds

\begin{tabular}{|c|c|c|}
\hline Variable & Herd A & Herd B \\
\hline Herd size, $\mathrm{n}$ & 66 to 128 & 255 to 289 \\
\hline Estrous detection rate, \% & 55 & 56 \\
\hline Conception rate, \% & 40 & 32 \\
\hline Voluntary waiting period, $\mathrm{d}$ & 60 & 60 \\
\hline $305 \mathrm{M}^{2}$ in lactation $1, \mathrm{~kg}$ & 8,897 & 7,994 \\
\hline $305 \mathrm{M}$ in lactation $2, \mathrm{~kg}$ & 10,189 & 9,589 \\
\hline $305 \mathrm{M}$ in lactation $\geq 3, \mathrm{~kg}$ & 10,358 & 9,998 \\
\hline Average days open (d) & 130 & 130 \\
\hline Heifer cost, ${ }^{3}$ Can $\$$ & \multicolumn{2}{|c|}{1,900} \\
\hline Cull price, ${ }^{4}$ Can $\$$ & \multicolumn{2}{|c|}{450} \\
\hline Milk price, Can $\$ / 100 \mathrm{~kg}$ & \multicolumn{2}{|c|}{40} \\
\hline Marginal feed costs/d, Can $\$$ & \multicolumn{2}{|c|}{10} \\
\hline Maintenance feed costs $/ \mathrm{d}, \mathrm{Can} \$$ & \multicolumn{2}{|c|}{2.50} \\
\hline Annual discount rate, $\%$ & \multicolumn{2}{|c|}{7} \\
\hline Culling risk in lactation $\geq 6, \%$ & \multicolumn{2}{|c|}{99} \\
\hline
\end{tabular}

${ }^{1}$ COWVAL (Cow Value) estimates of the value of a cow for a particular herd.

${ }^{2} 305 \mathrm{M}=$ estimated average 305-d cumulative milk yield.

${ }^{3}$ Heifer price is based on estimations by Lang (2003).

${ }^{4} \mathrm{Cull}$ price is based on the market price by Gencor (2006).

DELTA in the first month of lactation for cows in higher lactations was the difference between the last COWVAL of the dry period of the previous lactation and the first COWVAL within the new lactation. Cows with more than $80 \mathrm{~d}$ between DHI test dates (approximately 2 missed DHI tests in a row) were excluded from the analysis.

Disease data were extracted for disease events that occurred within $25 \mathrm{~d}$ before each test date, excluding events that occurred on the test date. The disease classes included were mastitis, feet and leg problems, metabolic and other diseases (e.g., milk fever, displaced abomasum, and traumatic reticuloperitonitis), reproductive disorders, and abortion. Disease data were recorded as a dichotomous variable (DISEASE): occurred (1) vs. not occurred (0). Each disease class could occur only once during the 25 -d period, even if multiple events of one disease class were recorded during the time between the 2 DHI tests. Additionally, for further analysis, the observations of the disease classes were combined per month to determine monthly disease presence or absence.

The variables COWVAL and DELTA were highly kurtotic, not normally distributed. The assumption of normality was tested using the Shapiro-Wilk, Cramervon Moses, Kolmogorov-Smirnov, and Anderson-Darling tests. All tests had a $P<0.001$. Consequently, the descriptive statistics report the median as the indicator of central tendency. Additionally, for simplicity, DELTA was dichotomized (DELTACUT) to reflect changes of a small or large magnitude between months, regardless of the starting point or the direction of the change. Small changes were all values of DELTA falling within the interquartile range (range between the 25th and 75th percentile), and large changes were all observations beyond those limits. The interquartile range was arbitrarily chosen to give a sufficiently large sample size in both groups (small and large DELTACUT). As an alternative example, if 2 standard deviations had been chosen as the cut point instead of the interquartile range, only $5 \%$ of the observations would have fallen into the large DELTACUT group. This small group of observations would have represented the extreme values at either end of the distribution, primarily influenced by outliers. This was not the aim of the investigation.

A GLM model was used for the evaluation of the predictors of DELTACUT, thereby addressing within cow clustering of data (i.e., repeated measures per cow). The model was created in SAS (SAS Institute Inc., Cary, NC) using the PROC GLIMMIX command. Based on the observed Akaike information criteria (AIC), a toeplitz-4 structure of DELTACUT over time was chosen, because this resulted in the lowest AIC compared with the other correlation structures offered by SAS and that converged. The tested covariance structures were: variance component (random effects), unstructured, compound symmetry, full toeplitz, toeplitz 2 to 6 , and first-order auto regressive [ar(1)] (Littell et al., 1996). The AIC was used as a criterion for comparing the fit of different error structures and penalized overfitting of the model. The model with the lowest AIC was the preferred model.

The timeline of observations throughout the lactation was measured in 30-d intervals from freshening (DSFSHCUT). Farm was included in the statistical analysis as a fixed effect, because cows from only 2 farms were studied. For the model, COWVAL was categorized (COWVALCUT) as below (0), within (1), or above (2) the interquartile range. This was done to assess whether the initial COWVAL could be used to predict the magnitude of change (DELTACUT). The change in the estimated 305-d milk production from DHI test to the next was categorized into 3 groups based on its interquartile range after 60 DIM (below $-220 \mathrm{~kg}$, between -220 and $160 \mathrm{~kg}$, or above $160 \mathrm{~kg}$ ) to enhance any observed effect of the estimated $305-\mathrm{d}$ milk production $(\mathbf{3 0 5 M})$. Only observations after 60 DIM were included in the GLIMMIX model. For the analysis, the values from the DHI test preceding DELTA were used, because they represented the starting point for the calculation of DELTA. To compare the estimates of the different groups of the categorical variables, the least squares means (lsmeans statement) were calculated in the GLIMMIX procedure. In the final model only factors with a $P$-value $\leq 0.05$ were retained. The final model included herd, lactation number, DSFSHCUT, COW- 
VALCUT, reproductive status, change in reproductive status between 2 milk tests (RPRODIFF), DISEASE, the second-order term DSFSHCUT ${ }^{2}$, and the 2-way interactions for DSFSHCUT interacting with reproductive status, 305M, COWVALCUT, and RPRODIFF. To compute the different probabilities for the different variables in the model at different time points throughout the lactation, the least squares means statement was adjusted accordingly.

A second GLIMMIX model was fitted to describe the relationship between certain factors and DELTACUT in the first 60 DIM. The change in the estimated 305M from one DHI test to the next was categorized into 3 groups based on its interquartile range in the first 60 DIM (below $-3,680 \mathrm{~kg}$, between $-3,681$ and $1,270 \mathrm{~kg}$, or above $1,270 \mathrm{~kg}$ ) to enhance any observed effect of the estimated $305 \mathrm{M}$. In the final model only factors with a $P$-value $\leq 0.05$ were retained. The final model included herd, lactation number, change in $305 \mathrm{M}$, DISEASE, and COWVALCUT.

To follow COWVAL within cows after falling below the baseline value of $\$ 0$, COWVAL was categorized into 3 groups: $\leq \$ 0, \$ 1$ to $\$ 499$, and $\geq \$ 500$. The boundary of $\$ 500$ was arbitrarily chosen because it was near the 10th percentile of all observed COWVAL observations and represented a distinct distance from the baseline. Only cows that had at least 1 COWVAL $\leq \$ 0$ were included in this analysis. The starting point for this analysis was the first test in which COWVAL dropped to $\leq \$ 0$. The highest subsequent category of the COWVAL (remained below $\$ 0$, rose to $\$ 1$ to $\$ 499$, or rose to $\geq \$ 500$ ) was recorded. This analysis was conducted including all observations over the whole lactation, and then separately, including only observations after 60 DIM.

The statistical and descriptive analyses were carried out utilizing the SAS software package (SAS 9.1 TS, SAS for Windows, SAS Institute); SPSS software (release 12.0.2, 2004, SPSS for Windows, SPSS, Chicago, IL) was used for the generation of the graphs.

\section{RESULTS}

\section{Description of COWVAL and DELTA}

The data included 20,071 observations from 1,463 cows eligible for analysis. The observed values for COWVAL in each herd and in total are presented in Table 2. The observed range in COWVAL (within each of the 2 herds) was comparable between herds: $\$ 7,787$ and $\$ 7,629$ in herds A and B, respectively. Overall, the highest and lowest observed COWVAL estimates were $\$ 6,382$ and $-\$ 2,298$, respectively. Fifty percent of all cows had a COWVAL between $\$ 1,049$ and $\$ 2,038$. Only $2.9 \%$ of all observations $(n=591)$ had a COWVAL $\leq \$ 0$ (Table 2).
Table 2. Descriptive statistics for COWVAL ${ }^{1}$ in the 2 Ontario dairy herds

\begin{tabular}{lrrr}
\hline $\begin{array}{l}\text { COWVAL } \\
\text { (Can } \$)\end{array}$ & Herd A & Herd B & Total \\
\hline $\mathrm{n}$ & 14,410 & 5,661 & 20,071 \\
Mean & 1,537 & 1,537 & 1,537 \\
SD & 793 & 838 & 806 \\
Minimum & $-2,298$ & $-1,247$ & $-2,298$ \\
25th percentile & 1,048 & 1,049 & 1,049 \\
Median & 1,547 & 1,545 & 1,546 \\
75th percentile & 2,044 & 2,022 & 2,038 \\
Maximum & 5,489 & 6,382 & 6,382 \\
Skewness & -0.1 & 0.6 & 0.1 \\
Kurtosis & 0.4 & 3.4 & 1.4 \\
$\% \leq$ Can $\$ 0$ & 2.8 & 3.2 & 2.9 \\
\hline
\end{tabular}

${ }^{1}$ COWVAL (Cow Value) estimates of the value of a cow for a particular herd.

The DELTA variable followed a similar pattern in both herds (Table 3). Half of the observed DELTA were between $-\$ 253$ and $\$ 176$. Still, large values and heavy tails led to a high level of kurtosis. The overall range in observed DELTA was $\$ 11,984$. Within herd A, the range in DELTA was $\$ 11,984$, and within herd B it was $\$ 9,160$ (Table 3).

\section{Lactation}

Figure 1 shows that the range of COWVAL was greatest in the first 30 DIM and decreased gradually throughout the rest of the lactation. This held true for all parity groups. Figure 2 shows the COWVAL estimates for lactations $1,2,3$, and $\geq 4$ over the first 120 DIM. There were between 104 and 674 observations for each month of lactation (e.g., 0 to 30 DIM in lactation 1). Visually, it appeared that an increase in the lactation number resulted in a lower median COWVAL (Figure 2 ), but the greatest median COWVAL were seen at the beginning of lactation 2 (31 to 60 DIM). For all lactations, the median COWVAL increased from the

Table 3. Descriptive statistics of DELTA $^{1}$ in the 2 Ontario dairy herds

\begin{tabular}{lrrr}
\hline $\begin{array}{l}\text { DELTA } \\
\text { (Can } \$)\end{array}$ & Herd A & Herd B & Total \\
\hline $\mathrm{n}$ & 13,275 & 5,333 & 18,608 \\
Mean & -37 & -12 & -30 \\
$\mathrm{SD}$ & 606 & 810 & 671 \\
Minimum & $-7,787$ & $-5,051$ & $-7,787$ \\
25th percentile & -255 & -248 & -253 \\
Median & -29 & 9 & -19 \\
75th percentile & 157 & 224 & 176 \\
Maximum & 4,197 & 4,109 & 4,197 \\
Skewness & 0.4 & -0.4 & 0.1 \\
Kurtosis & 10.9 & 8.1 & 10.3 \\
\hline
\end{tabular}

${ }^{1}$ Difference of COWVAL (Cow Value estimates of a cow's value for a particular herd) between 2 consecutive DHI tests. 


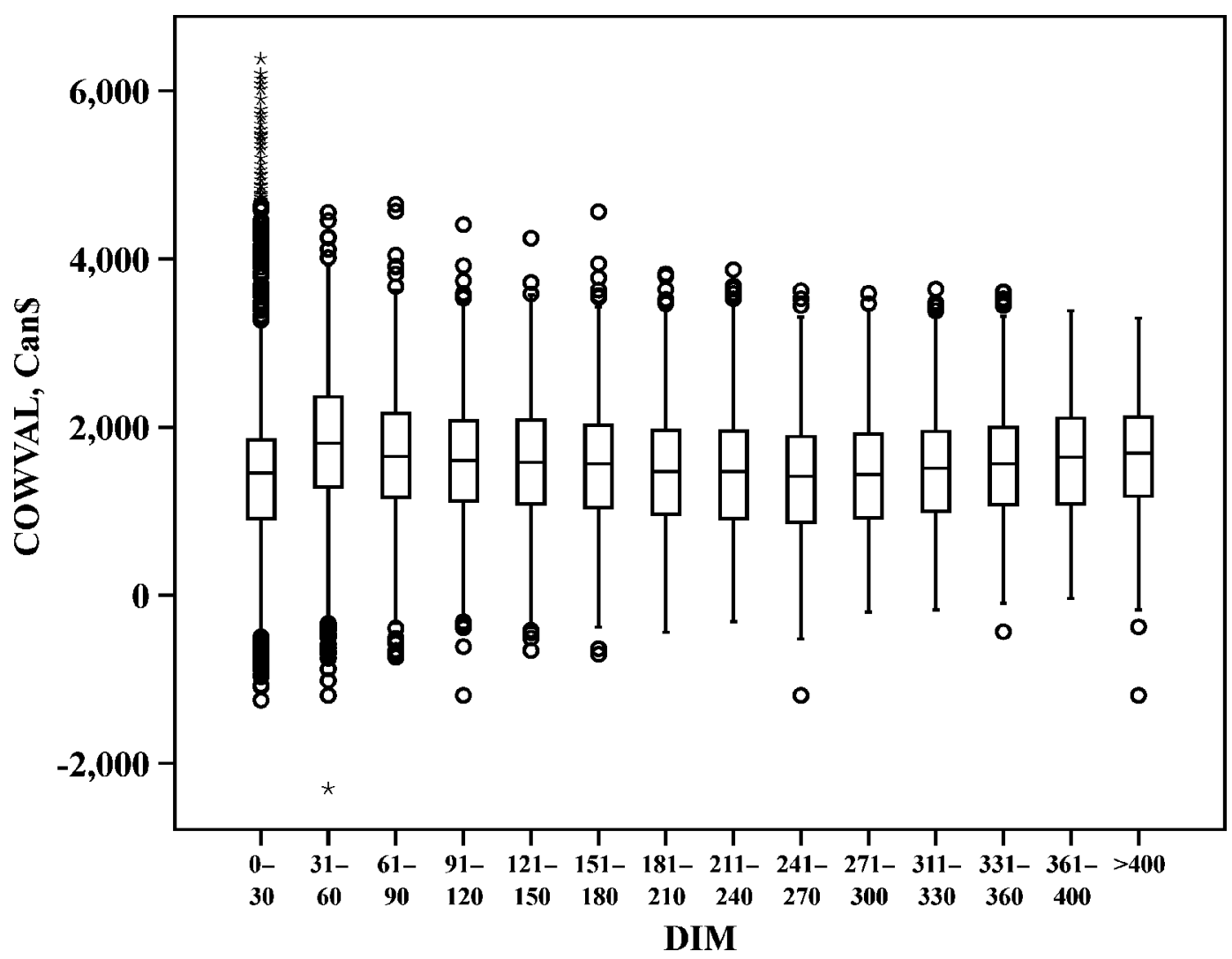

Figure 1. Box-plot of COWVAL (Cow Value estimates of a cow's value for a particular herd) over DIM; $\bigcirc=$ outlier, * $=$ extreme value.

first to the second month, then declined until the eighth month and finally increased again in the later days of the lactation (data not shown). Comparing DELTA across lactations revealed that the biggest drop in the median COWVAL occurred from peak to 241 to 270 DIM in lactation 2. The cows in lactation 1 showed the least change in the median COWVAL over this period (results not shown).

The variation in COWVAL and DELTA was greatest in the early stages of lactation. The greatest variation in DELTA was observed in the second month of lactation. Starting in the third month of lactation the range in DELTA values decreased (Figure 3). In that period, the majority (95\%) of the 305M estimates dropped; that is, the change in $305 \mathrm{M}$ was negative, and ranged between $-15,590$ and $5,420 \mathrm{~kg}$. Cows that dropped considerably (more than $3,690 \mathrm{~kg}$ ) in their $305 \mathrm{M}$ were 2.8 times as likely to have a large DELTACUT as cows with large positive changes in their 305M (Table 4). Table 4 shows the effect of factors resulting in large DELTACUT in the first 60 DIM. Disease events had little influence on the occurrence of large DELTACUT in this period [upper 95\% confidence limit of odds ratio was close to $1 ; P=0.0250$; Table 4]. Yet, a comparison of the 4 lactations showed that cows in lactations $\geq 4$ had the lowest odds to show large DELTACUT, whereas cows in the second lactation had the greatest odds to show a large DELTACUT (Table 4).

Between d 61 and 271 of lactation, DELTA was predominantly negative, which is consistent with the previously described behavior of COWVAL and changes in 305M. Moreover, DELTA had positive values at the end of lactation (Figure 3). Throughout the lactation, cows in herd A were less prone to large DELTACUT than cows in herd $\mathrm{B}$ (odds ratio $\leq 0.8 ; P<0.0001$; Tables 4 and 5).

Tables 5 and 6 present the factors associated with large DELTACUT after 60 DIM. Again, cows in lactations $\geq 4$ were less likely to have a large DELTACUT than cows in lactations 1 to $3(P<0.001$; Table 5$)$.

Furthermore, throughout the lactation, cows with a small COWVALCUT (i.e., COWVAL below the interquartile range) were most likely to show large DELTACUT compared with cows with medium or large COWVALCUT (Table 6). Cows with a small COWVALCUT were in higher lactations (lactation number: 3.1 vs. 1.9 , respectively; $P<0.001)$ and seemed to have a lower estimated $305 \mathrm{M}$ (9,339 vs. $10,456 \mathrm{~kg}$, respec- 


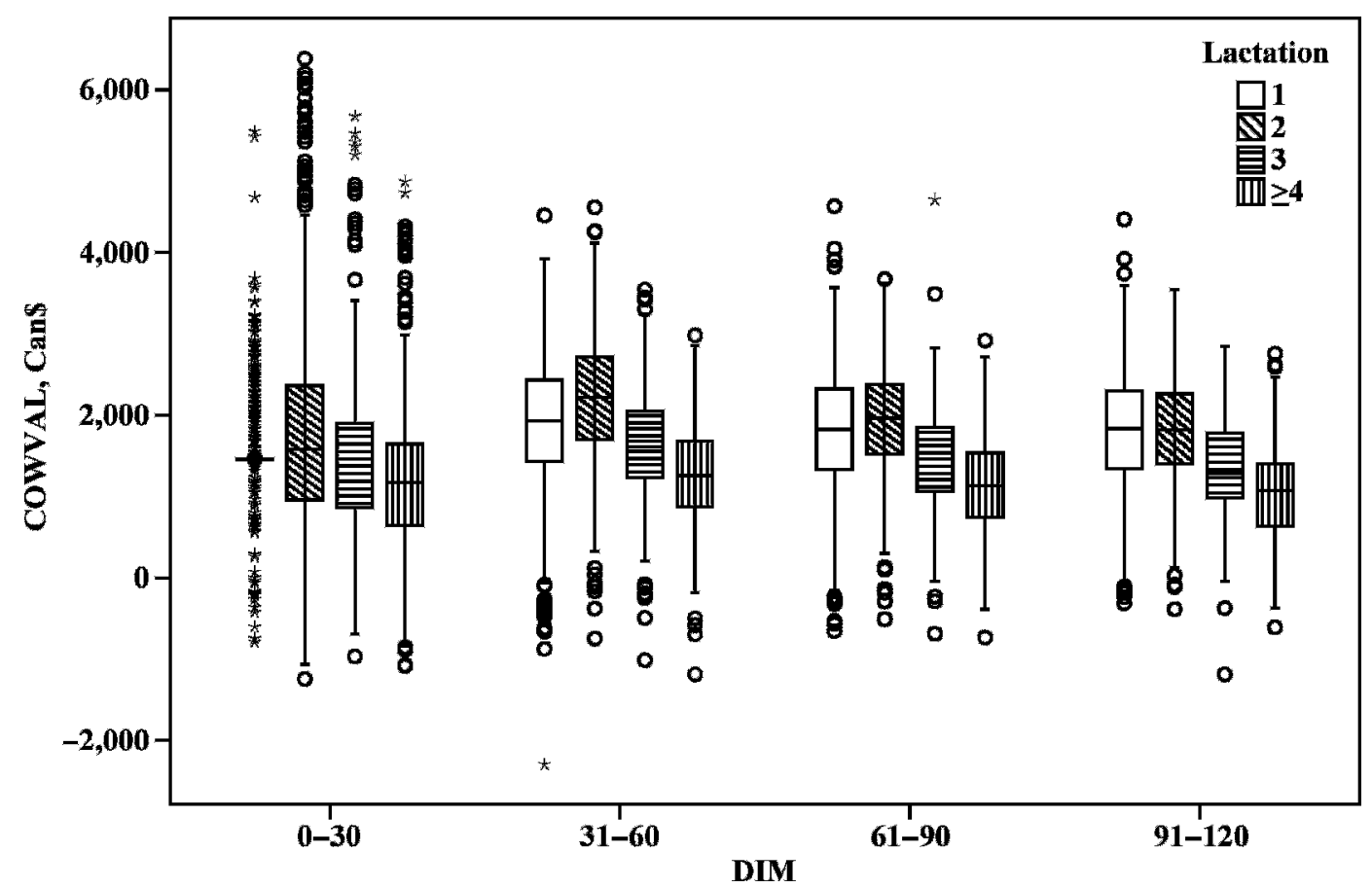

Figure 2. Box-plot of COWVAL (Cow Value estimates of a cow's value for a particular herd) over the first 120 DIM in lactations 1 through $\geq 4 ; O=$ outlier, $*$ = extreme value.

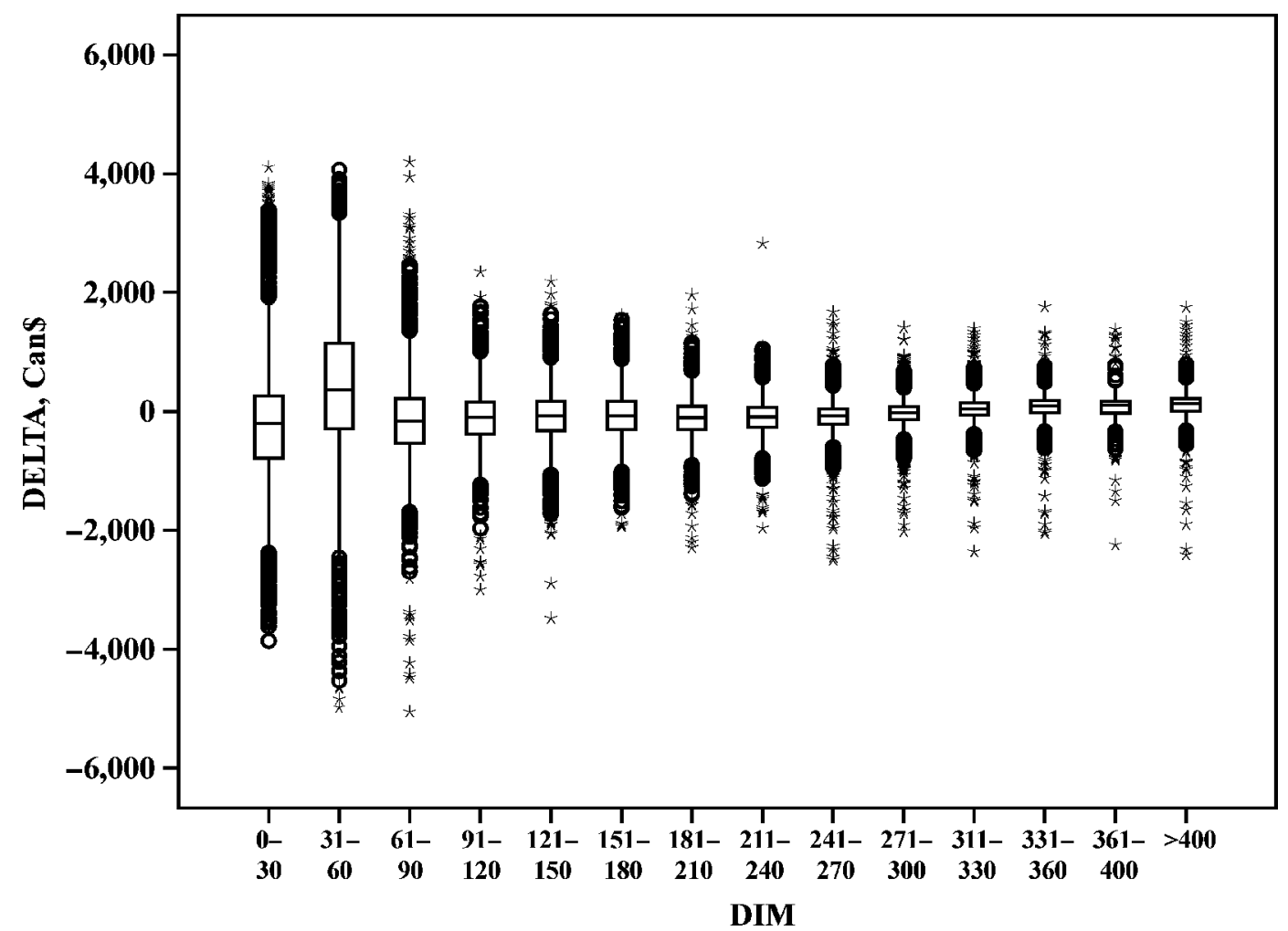

Figure 3. Box-plot showing DELTA, the difference in COWVAL (Cow Value estimates of a cow's value for a particular herd) between 2 consecutive DHI tests; $\bigcirc=$ outlier, * = extreme value. 
Table 4. Variables affecting the occurrence of small or large ${ }^{1}$ monthly changes in COWVAL (DELTACUT, Can\$) in the first 60 DIM

\begin{tabular}{|c|c|c|c|c|}
\hline Variable $^{2}$ & Odds ratio $(\mathrm{OR})$ & $95 \% \mathrm{CL}^{3}$ of $\mathrm{OR}$ & Estimate \pm SEM & $P$-value \\
\hline Intercept & - & - & $1.538 \pm 0.224$ & $<0.0001$ \\
\hline Herd A & 0.6 & $0.41-0.74$ & $-0.597 \pm 0.154$ & $<0.0001$ \\
\hline Herd B & Reference & & & \\
\hline Lactation 1 & 2.5 & $1.69-3.55$ & $0.897 \pm 0.188$ & $<0.0001$ \\
\hline Lactation 2 & 5.2 & $3.65-7.53$ & $1.657 \pm 0.184$ & $<0.0001$ \\
\hline Lactation 3 & 1.6 & $1.15-2.11$ & $0.443 \pm 0.154$ & 0.0042 \\
\hline Lactation $\geq 4$ & Reference & & & \\
\hline \multicolumn{5}{|l|}{$305 \mathrm{M}$ change ${ }^{4}$} \\
\hline$\leq-3,680 \mathrm{~kg}$ & 2.8 & $1.92-3.98$ & $1.017 \pm 0.186$ & $<0.0001$ \\
\hline$-3,679$ to $1,270 \mathrm{~kg}$ & $\begin{array}{c}1.0 \\
\text { Beference }\end{array}$ & $0.75-1.26$ & $-0.030 \pm 0.133$ & 0.8241 \\
\hline$>1,270 \mathrm{~kg}$ & Reference & & & \\
\hline DISEASE $^{5}$ & 0.7 & $0.49-0.95$ & $-0.37 \pm 0.168$ & 0.0250 \\
\hline \multicolumn{5}{|l|}{ COWVALCUT $^{6}$} \\
\hline Small & 0.9 & $0.60-1.46$ & $-0.063 \pm 0.226$ & 0.7808 \\
\hline Medium & 0.5 & $0.37-0.74$ & $-0.653 \pm 0.176$ & 0.0002 \\
\hline Large & Reference & & & \\
\hline
\end{tabular}

\footnotetext{
${ }^{1}$ Small and large $=$ values within or outside of the interquartile range of DELTA, respectively. The interquartile range of DELTA is $-\$ 253$ to $\$ 176$. DELTA is the difference of COWVAL (Cow Value estimates of a cow's value for a particular herd) between 2 consecutive DHI tests. All values in Canadian dollars.

${ }^{2}$ The statistical procedure used was PROC GLIMMIX. The correlation structure of the model was toeplitz4 ; the correlation coefficient estimate was 0.1227 (SEM 0.05257).

${ }^{3} 95 \%$ confidence limits.

${ }^{4}$ Change in the estimated cumulative $305-\mathrm{d}$ milk production (305M) between monthly DHI tests.

${ }^{5}$ DISEASE describes whether a disease event occurred (1) or not (0) in the last $25 \mathrm{~d}$.

${ }^{6}$ COWVALCUT is the COWVAL (Can $\$$ ) - categorized into small $(\leq \$ 1,049)$, medium $(\$ 1,050$ to $\$ 2,038)$, and large $(>2,038)$. The cut points were based on the interquartile range of COWVAL.
}

tively) than cows with medium or high COWVALCUT. Nonetheless, the dependence of the data (i.e., repeated measures per cow) impedes a comparison of these averages.

Besides these factors, the reproductive status of cows, its change, and changes in the estimated $305 \mathrm{M}$ had a large influence on DELTACUT (Table 6). Cows that were open (past the voluntary waiting period and known not pregnant) had the greatest odds of expressing large DELTACUT (assuming that DSFSHCUT = 0; $P<0.001)$. Nevertheless, as lactation progressed, the probability for large DELTACUT decreased for open cows and became significantly lower than for pregnant or bred cows $(P<0.001)$. On the other hand, the probability of large DELTACUT increased throughout lactation for bred cows and those that changed in their reproductive status (Table 6).

\section{Behavior of COWVAL After Dropping to $<\$ 0$}

The COWVAL of 440 cows $(30.1 \%$ of all cows in the final data set) dropped to or below the baseline of $\$ 0$ on at least one occasion during their productive lives. When these cows were followed for the remainder of their productive lives, $25.7 \%(\mathrm{n}=113)$ remained below the baseline of $\$ 0$, whereas $69.1 \%(\mathrm{n}=304)$ had a COWVAL of $>\$ 500$ at least once later in their lives. Approxi- mately one-third of the 440 cows $(34.1 \%, \mathrm{n}=150)$ stayed in the herd for at least 1 additional lactation after reaching $\$ 0$. Of those 150 cows, 33 stayed for a third, and 19 for a fourth lactation.

A similar analysis was conducted using only cows with COWVAL dropping to or below $\$ 0$ after 60 DIM. Given these criteria, the COWVAL of 171 cows were available for analysis. The COWVAL of $54.4 \%$ of these cows $(\mathrm{n}=93)$ remained below $\$ 0$, whereas the COWVAL of $31.6 \%$ of the 171 cows $(n=54)$ increased at time to $\geq \$ 500$. These observations were independent of the lactation number of the cows $(P>0.05)$ when their COWVAL reached the baseline. Again, a few cows ( $\mathrm{n}=$ 16) started a second, third $(\mathrm{n}=9)$, or fourth $(\mathrm{n}=3)$ lactation subsequently.

Cows whose COWVAL dropped to or below $\$ 0$ in the first 60 DIM were $38.0 \mathrm{~d}$ postfreshening, whereas cows above the baseline were 29.6 DIM $(P<0.0001)$. Additionally, cows below the baseline had greater 305M than cows that stayed above the baseline: 8,229 and $7,748 \mathrm{~kg}$, respectively, and appeared to be slightly younger: 2.1 vs. 2.3 lactations, respectively.

\section{Reproductive Status of the Cow}

The reproductive status of the cows had an impact on their COWVAL (Figure 4). When following the COW- 
Table 5. Variables affecting the occurrence of small or large ${ }^{1}$ monthly changes in COWVAL (DELTACUT, Can\$) after 60 DIM

\begin{tabular}{|c|c|c|c|c|}
\hline Variable $^{2}$ & $\begin{array}{l}\text { Odds ratio } \\
\text { (OR) }\end{array}$ & $\begin{array}{c}95 \% \mathrm{CL}^{3} \\
\text { of OR }\end{array}$ & Estimate \pm SEM & $P$-value \\
\hline Intercept & - & - & $-0.062 \pm 0.208$ & 0.7653 \\
\hline Herd A & 0.8 & $0.7-0.9$ & $-0.247 \pm 0.045$ & $<0.0001$ \\
\hline Herd B & Reference & & & \\
\hline Lactation 1 & 1.2 & $1.1-1.4$ & $0.216 \pm 0.065$ & 0.0009 \\
\hline Lactation 2 & 1.4 & $1.3-1.6$ & $0.349 \pm 0.065$ & $<0.0001$ \\
\hline Lactation 3 & 1.3 & $1.2-1.5$ & $0.278 \pm 0.067$ & $<0.0001$ \\
\hline Lactation $\geq 4$ & Reference & & & \\
\hline DSFSHCUT $^{4}$ & 1.1 & $0.9-1.2$ & $0.067 \pm 0.036$ & 0.0601 \\
\hline \multicolumn{5}{|l|}{ COWVALCUT $^{5}$} \\
\hline Small & 0.4 & $0.3-0.5$ & $-0.979 \pm 0.144$ & $<0.0001$ \\
\hline Medium & 0.5 & $0.4-0.6$ & $-0.730 \pm 0.116$ & $<0.0001$ \\
\hline Large & Reference & & & \\
\hline \multicolumn{5}{|l|}{$305 \mathrm{M}$ change ${ }^{6}$} \\
\hline$<-220 \mathrm{~kg}$ & 5.7 & $4.3-7.7$ & $1.751 \pm 0.149$ & $<0.0001$ \\
\hline-220 to $160 \mathrm{~kg}$ & 0.2 & $0.2-0.3$ & $-1.509 \pm 0.145$ & $<0.0001$ \\
\hline$>160 \mathrm{~kg}$ & Reference & & & \\
\hline \multicolumn{5}{|l|}{ Reproductive status } \\
\hline Open & 5.7 & $4.1-7.9$ & $1.745 \pm 0.162$ & $<0.0001$ \\
\hline Pregnant & 1.1 & $0.7-1.5$ & $0.064 \pm 0.188$ & 0.7339 \\
\hline Bred & Reference & & & \\
\hline RPRODIFF $^{7}$ & 5.0 & $3.7-6.8$ & $1.613 \pm 0.153$ & $<0.0001$ \\
\hline DISEASE $^{8}$ & 1.4 & $1.0-1.9$ & $0.313 \pm 0.154$ & $<0.0008$ \\
\hline DSFSHCUT $\times$ DSFSHCUT $^{9}$ & 1.0 & $1.0-1.0$ & $0.015 \pm 0.002$ & $<0.0001$ \\
\hline \multicolumn{5}{|c|}{ DSFSHCUT $\times$ Reproductive status } \\
\hline Open & 0.7 & $0.7-0.7$ & $-0.365 \pm 0.287$ & $<0.0001$ \\
\hline Pregnant & 0.8 & $0.9-0.9$ & $-0.111 \pm 0.024$ & $<0.0001$ \\
\hline Bred & Reference & & & \\
\hline \multicolumn{5}{|l|}{ DSFSHCUT $\times 305 \mathrm{M}$ change } \\
\hline$\leq-220 \mathrm{~kg}$ & 0.8 & $0.8-0.8$ & $-0.209 \pm 0.021$ & $<0.0001$ \\
\hline-220 to $160 \mathrm{~kg}$ & 1.1 & $1.0-1.1$ & $0.073 \pm 0.019$ & 0.0001 \\
\hline$>160 \mathrm{~kg}$ & Reference & & & \\
\hline \multicolumn{5}{|l|}{ DSFSHCUT $\times$ COWVALCUT } \\
\hline Small & 1.2 & $1.1-1.2$ & $0.166 \pm 0.016$ & $<0.0001$ \\
\hline Medium & 1.1 & $1.1-1.1$ & $0.086 \pm 0.014$ & $<0.0001$ \\
\hline Large & Reference & & & \\
\hline \multicolumn{4}{|l|}{$\mathrm{DSFSH} \times \mathrm{RPRODIFF}$} & $<0.0001$ \\
\hline No & 0.7 & $0.7-0.7$ & $-0.351 \pm 0.024$ & $<0.0001$ \\
\hline Yes & Reference & & & $<0.0001$ \\
\hline
\end{tabular}

${ }^{1}$ Small and large $=$ values within or outside of the interquartile range of DELTA, respectively. The interquartile range of DELTA is $-\$ 253$ to $\$ 176$. DELTA is the difference of COWVAL (Cow Value estimates of a cow's value for a particular herd) between 2 consecutive DHI tests. All values in Canadian dollars.

${ }^{2}$ The statistical procedure used was PROC GLIMMIX. The correlation structure of the model was toeplitz4; the correlation coefficient estimate is 0.02299 (SEM 0.00956).

${ }^{3} 95 \%$ confidence limits.

${ }^{4}$ DSFSHCUT $=\mathrm{d}$ since freshening, in 30-d intervals.

${ }^{5}$ COWVALCUT is the COWVAL (Can $\$$ ) - categorized into small ( $\left.\leq \$ 1,049\right)$, medium $(\$ 1,050$ to $\$ 2,038)$, and large $(>\$ 2,038)$. The cut points were based on the interquartile range of COWVAL.

${ }^{6}$ Change in the estimated cumulative $305-\mathrm{d}$ milk production $(305 \mathrm{M})$ between monthly DHI tests. The categories are based on the confidence limits.

${ }^{7} \mathrm{RPRODIFF}=$ dichotomous variable describing whether a change in the reproductive status of the cow occurred (1) or not (0) during the month. Change may be from open to bred, bred to pregnant, or pregnant to open.

${ }^{8}$ DISEASE describes whether a disease event occurred (1) or not (0) in the last $25 \mathrm{~d}$.

${ }^{9}$ Interaction term between 2 variables is indicated by $\times$.

VAL of open, bred, and pregnant cows over lactation, differences were observed between the COWVAL of the different reproductive status groups throughout the lac- tation (Figure 4). Except at the beginning of the lactation, open cows had the lowest COWVAL and bred cows had lower COWVAL than pregnant cows (Figure 4). As 
Table 6. Probability for large ${ }^{1}$ DELTACUT (Can\$) at various DIM given the reproductive status, difference in predicted cumulative 305-d production (305M change), COWVALCUT, and RPRODIFF

\begin{tabular}{|c|c|c|c|c|}
\hline \multirow{2}{*}{$\begin{array}{l}\text { Variable in } \\
\text { interaction with } \\
\text { DSFSHCUT }^{2,3}\end{array}$} & \multicolumn{4}{|c|}{ DIM } \\
\hline & 91 to 120 & 181 to 210 & 271 to 300 & 361 to 390 \\
\hline \multicolumn{5}{|c|}{ Reproductive status } \\
\hline Open & 0.68 & 0.45 & 0.35 & 0.29 \\
\hline Pregnant & 0.52 & 0.47 & 0.56 & 0.68 \\
\hline Bred & 0.61 & 0.64 & 0.79 & 0.89 \\
\hline \multicolumn{5}{|l|}{$305 \mathrm{M}$ change ${ }^{4}$} \\
\hline$\leq-220 \mathrm{~kg}$ & 0.81 & 0.65 & 0.59 & 0.55 \\
\hline-220 to $160 \mathrm{~kg}$ & 0.33 & 0.34 & 0.48 & 0.65 \\
\hline$>160 \mathrm{~kg}$ & 0.63 & 0.58 & 0.66 & 0.76 \\
\hline \multicolumn{5}{|l|}{ COWVALCUT $^{5}$} \\
\hline Small & 0.59 & 0.56 & 0.67 & 0.79 \\
\hline Medium & 0.57 & 0.48 & 0.54 & 0.63 \\
\hline Large & 0.66 & 0.52 & 0.51 & 0.53 \\
\hline \multicolumn{5}{|l|}{ RPRODIFF $^{6}$} \\
\hline No & 0.63 & 0.42 & 0.35 & 0.31 \\
\hline Yes & 0.58 & 0.62 & 0.78 & 0.89 \\
\hline
\end{tabular}

\footnotetext{
${ }^{1}$ Small and large $=$ values within or outside of the interquartile range of DELTA, respectively. The interquartile range of DELTA is $-\$ 253$ to $\$ 176$. DELTA is the difference of COWVAL (Cow Value estimates of a cow's value for a particular herd) between 2 consecutive DHI tests. All values in Canadian dollars.

${ }^{2}$ The statistical procedure used was PROC GLIMMIX. The correlation structure of the model was toeplitz4; the correlation coefficient estimate is 0.02299 (SEM 0.00956).

${ }^{3}$ DSFSHCUT $=\mathrm{d}$ since freshening, in 30-d intervals.

${ }^{4}$ Change in the estimated cumulative $305-\mathrm{d}$ milk production $(305 \mathrm{M})$ between monthly DHI tests. The categories are based on the confidence limits.

${ }^{5}$ COWVALCUT is the COWVAL (Can $\$$ ) - categorized into small ( $\left.\leq \$ 1,049\right)$, medium $(\$ 1,050$ to $\$ 2,038)$, and large $(>\$ 2,038)$. The cut points were based on the interquartile range of COWVAL.

${ }^{6} \mathrm{RPRODIFF}$ = dichotomous variable describing whether a change in the reproductive status of the cow occurred (1) or not (0) during the month. Change may be from open to bred, bred to pregnant, or pregnant to open.
}

the lactation progressed, more of the open cows were either bred or became cows that the farmer actively decided not to breed (i.e., "do not breed"). Furthermore, the fraction of bred cows declined, whereas the proportion of the pregnant cows increased.

At the beginning of a new lactation (fresh), COWVAL had the largest standard deviation $(\$ 1,035)$ compared with COWVAL in the other reproductive groups (Figure 5). Cows that were considered open showed a slightly lower COWVAL than cows that were fresh, bred, or pregnant (pregnant and dry). In contrast, cows that were designated as "do not breed" had the lowest COWVAL (median $\$ 180$, minimum $-\$ 2,298$, maximum $\$ 4,304$; Figure 5).

\section{Disease Events}

A total of 734 disease events were entered. Comparing months with $(\mathrm{n}=709)$ and without $(\mathrm{n}=19,362)$ disease events showed that the occurrence of disease within $25 \mathrm{~d}$ before a DHI test did not significantly influence COWVAL. The observed median and maximum COWVAL of diseased cows appeared slightly lower than the COWVAL of cows without reported diseases (Table 7).

Separating the diseases into different disease classes showed that the lowest median COWVAL was in cows that aborted, followed by those that experienced a disease in the "metabolic and other diseases" group (Table 7).

The comparison of DELTA in months with vs. months without disease events showed that the occurrence of disease influenced the observed DELTA. Cows with disease events dropped more in their COWVAL than did cows without diseases (Table 7). Additionally, the interquartile range was wider for DELTA in the diseased cows.

The division into the different disease categories revealed that the median DELTA of cows with "reproductive diseases" was comparable with the DELTA of disease-free months (Table 7). The lowest median DELTA was observed after abortions, followed by lameness, mastitis, and metabolic and other diseases.

\section{Culling}

The farmers recorded various culling reasons $(\mathrm{n}=$ 749). The removal reasons were reproductive failure 


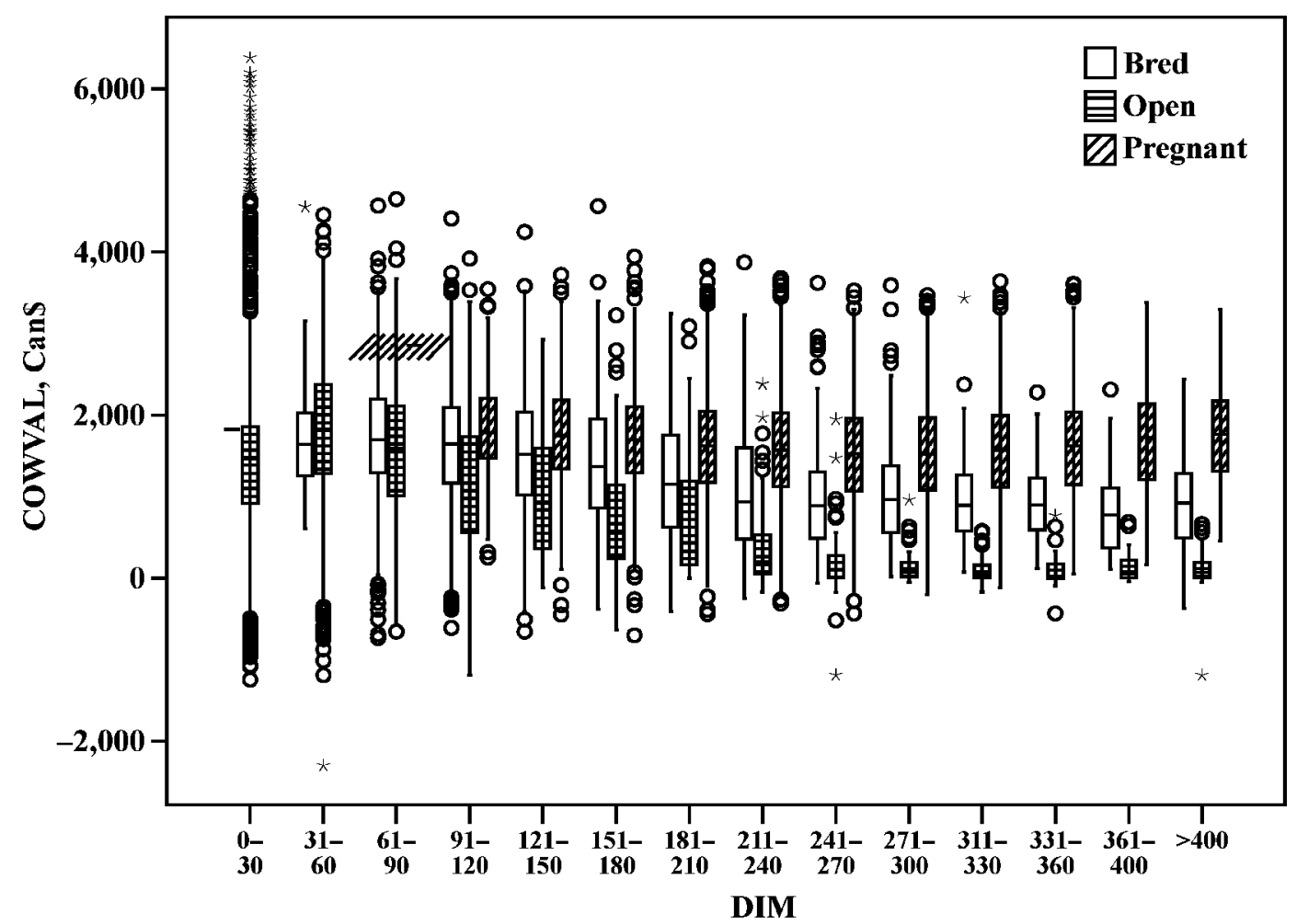

Figure 4. Box-plot of COWVAL (Cow Value estimates of a cow's value for a particular herd) over DIM by reproductive status (open, bred, or pregnant); $\bigcirc=$ outlier, ${ }^{*}=$ extreme value.

(29.3\%), feet/leg problems (15.8\%), udder (7.8\%), mastitis $(10.8 \%)$, death $(17.2 \%)$, low production $(9.1 \%)$, dairy sales $(0.9 \%)$, injury $(3.1 \%)$, sick $(4.9 \%)$, temperament $(0.4 \%)$, slow milker $(0.5 \%)$, and no reason $(0.1 \%)$. Figure 6 shows the distribution of the last recorded COWVAL before the cull event by removal reason including the data of both farms. The COWVAL of cows that were culled had a median value of $\$ 761$ with a range between $-\$ 1,190$ and $\$ 5,781$. Cows culled for low production and reproductive failure had the lowest COWVAL. In contrast, cows that were sold to other dairies, that died, or that were sick had the highest median values at exit from the herd.

\section{DISCUSSION}

The size and production level of the 2 selected herds was slightly above the average dairy herd in southwestern Ontario. Herd size ranged from 66 to 128 and from 255 to 289 cows, respectively, whereas the average dairy herd in Ontario has 55 cows (DFO, 2006). Additionally, the cumulative milk production per cow per $305 \mathrm{~d}(9,815$ and $9,194 \mathrm{~kg}$, respectively) was higher on the selected farms than the average production of 8,235 $\mathrm{kg}$ per cow per lactation in southwestern Ontario (DFO, 2006). These factors and the fact that only 2 farms were included in the study limit a generalization of the results and conclusions.

The observed values of COWVAL and DELTA followed distinct patterns. Within the first 60 to 90 DIM, COWVAL was unstable and highly variable even within a single cow. These high fluctuations between 2 consecutive DHI tests led to large values in DELTA for that period. Yet, as more DHI tests became available for a cow and the risk of changes in reproductive status or estimated milk production decreased, the more stable COWVAL became. Consequently, DELTA became more stable and smaller. These observations were supported by an odds ratio $<1$ for DSFSHCUT. These observations were true regardless of the lactation number of the animal, although cows in lactation $\geq 4$ were less likely to express large DELTACUT (1) than cows in lactation 1,2 , and 3 . This is probably due to more production information from previous lactations in older cows.

The COWVAL variable was influenced by the pregnancy status of the cow, particularly later in lactation. The relative value of a cow in a dairy herd increases when she transitions from being open to being pregnant. Conversely, her value usually declines if she loses a pregnancy. A pregnancy was more valuable at a later stage in lactation and pregnancy than at the beginning. Cows aborting late in the lactation and pregnancy will 


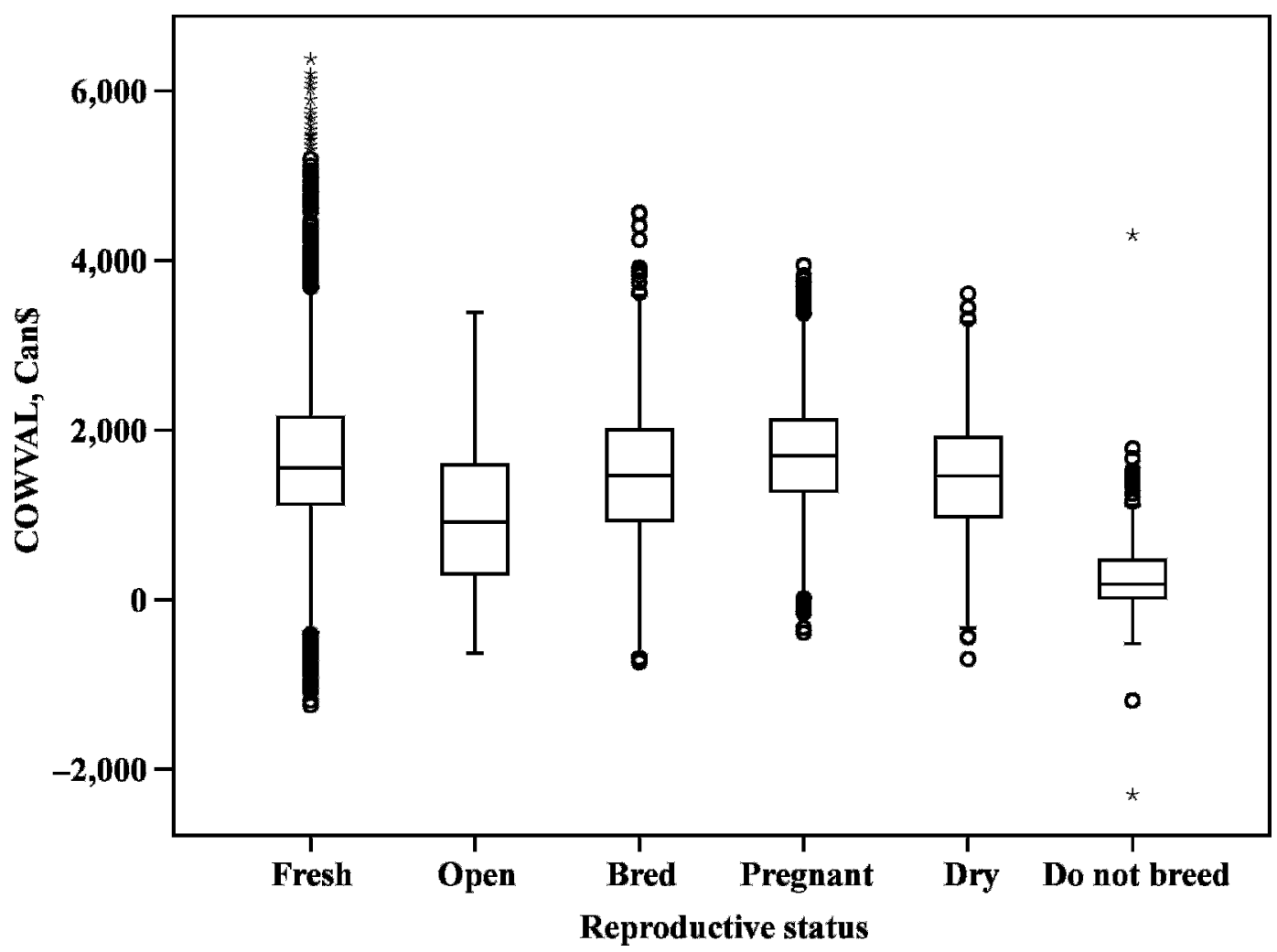

Figure 5. Box-plot of COWVAL (Cow Value estimates of a cow's value for a particular herd) for different reproductive status; $\bigcirc=$ outlier, $*$ = extreme value.

unlikely be bred again, and therefore, will be removed from the herd as open cows. Cows that were open and will no longer be bred ("do not breed") had the lowest average COWVAL, which was consistent with their status in the herd. They were most likely replaced once their production dropped below a certain level and a suitable replacement was available. For those cows that were still eligible for breeding, those classified open had the lowest COWVAL after the voluntary waiting period. The average COWVAL of fresh, bred, and pregnant

Table 7. Descriptive statistics of COWVAL ${ }^{1}$ and DELTA $^{2}$ (Can\$) for months with and without disease events and over different types of disease events

\begin{tabular}{|c|c|c|c|c|c|c|c|}
\hline Variable & Diseased & No disease & Mastitis & Lameness & $\begin{array}{l}\text { Metabolic } \\
\text { and other }\end{array}$ & $\begin{array}{l}\text { Reproductive } \\
\text { disease }\end{array}$ & Abortion \\
\hline \multicolumn{8}{|l|}{ COWVAL $(\mathrm{Can} \$)$} \\
\hline Median & 1,458 & 1,551 & 1,351 & 1,403 & 1,290 & 1,547 & 1,169 \\
\hline Minimum & $-1,079$ & $-2,289$ & $-1,079$ & -373 & $-1,079$ & $-1,079$ & -529 \\
\hline 25th percentile & 1,005 & 1,050 & 719 & 666 & 644 & 1,208 & 547 \\
\hline 75th percentile & 1,962 & 2,040 & 1,634 & 1,581 & 1,821 & 2,136 & 1,164 \\
\hline Maximum & 5,601 & 6,382 & 4,409 & 3,597 & 5,601 & 4,409 & 3,210 \\
\hline $\mathrm{n}$ & 709 & 19,362 & 172 & 29 & 48 & 443 & 42 \\
\hline \multicolumn{8}{|l|}{ DELTA $(\mathrm{Can} \$)$} \\
\hline Median & -131 & -17 & -195 & -201 & -188 & -37 & -257 \\
\hline Minimum & $-3,368$ & $-5,051$ & $-3,368$ & 1,113 & $-3,225$ & $-2,954$ & $-2,499$ \\
\hline 25th percentile & -554 & -246 & -564 & -443 & -954 & -502 & -647 \\
\hline 75th percentile & 244 & 175 & 26 & -39 & 117 & 435 & 111 \\
\hline Maximum & 4,197 & 4,109 & 2,649 & 1,384 & 3,631 & 4,197 & 674 \\
\hline $\mathrm{n}$ & 565 & 18,042 & 34 & 24 & 46 & 341 & 40 \\
\hline
\end{tabular}

${ }^{1}$ COWVAL (Cow Value) estimates of the value of a cow for a particular herd.

${ }^{2}$ Difference of COWVAL between 2 consecutive DHI tests. 


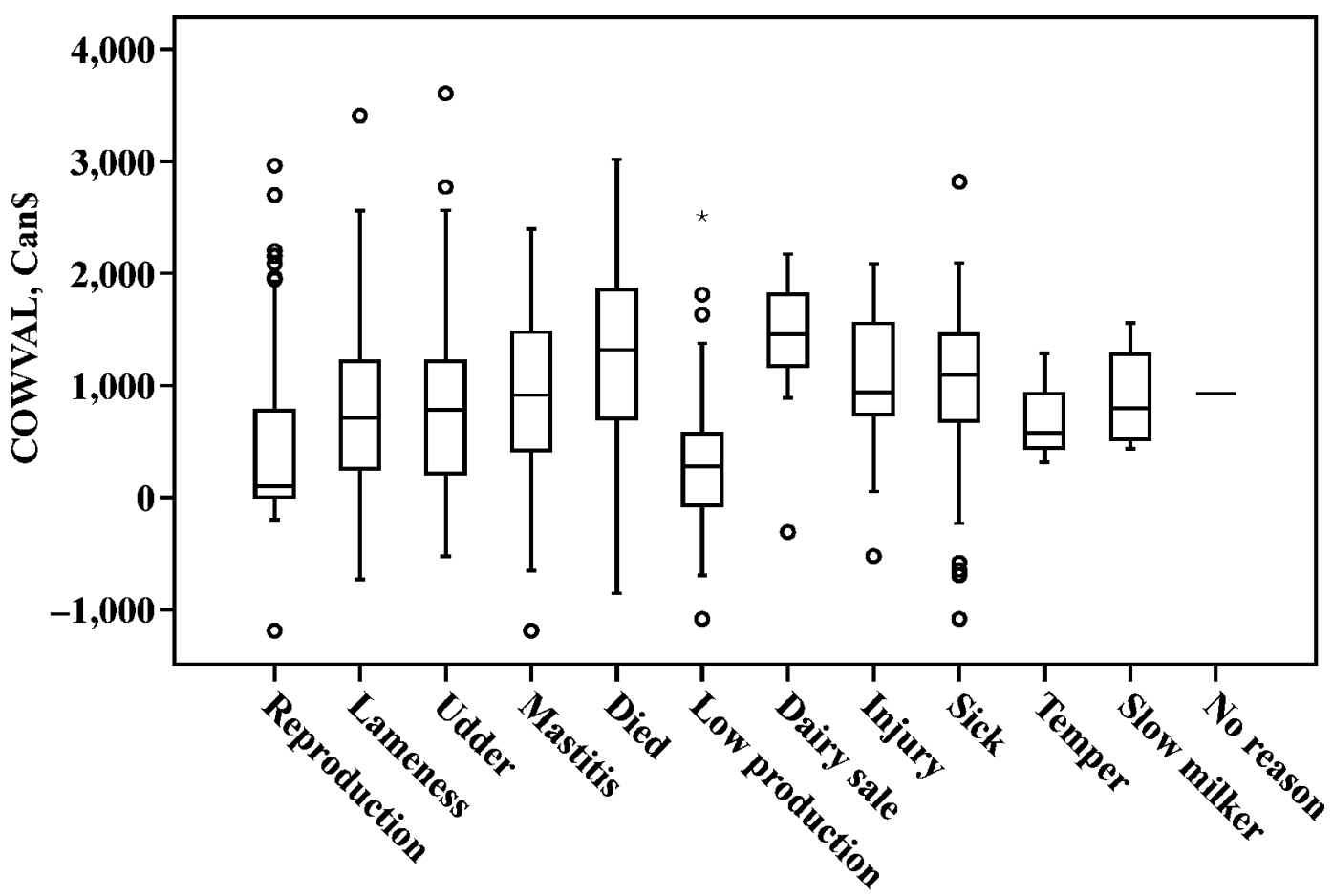

\section{Culling reason}

Figure 6. Box-plot of COWVAL (Cow Value estimates of a cow's value for a particular herd) at exiting of the herd for various culling reasons; $\bigcirc=$ outlier, $*$ = extreme value.

cows, including dry cows, was similar among these classes and was higher than that for open cows. Furthermore, changes in the reproductive status after 60 DIM led to large DELTACUT, which supports the importance of the pregnancy status in the calculation of COWVAL.

When followed over the lactation, COWVAL estimates formed a slightly sigmoid-shaped curve. The decrease in the middle of the lactation was most likely due to the combination of the decrease in milk production and uncertainties in the reproductive status of many cows. The majority of the cows at this stage of lactation were bred, but had an unknown pregnancy status and would not have been attributed the full value of pregnancy yet.

Later in the lactation, the reproductive status of the cow was more clearly established. The fraction of open cows became dominated by cows declared as "do not breed." This meant that their risk of changing their reproductive status was virtually zero, and a large DELTACUT was unlikely, as expressed in the low probabilities for large DELTACUT for open cows at the end of the lactation. On the other hand, the fraction of bred cows, which had the greatest risk of changing their reproductive status and thus COWVAL, was replaced with confirmed pregnant cows, which resulted in stability in COWVAL and smaller DELTA.

Cows that stay in the herd until the later stages of lactation are either more likely to enter a new lactation and to produce milk in the future, or they are producing enough milk to be profitable until their eventual removal. Consequently, the mean COWVAL increased in the later stages of lactation. Even after removing the observations from each culled cow's last lactation in the herd, COWVAL followed a sigmoid curve over DIM. This finding reinforced the fact that pregnancy status and stage of lactation highly influenced the COWVAL of cows. At the beginning of the lactation, the 305-d milk production estimate was not properly established. It was obvious that cows with medium and high COWVAL and large decreases or increases in 305M between 2 tests were more likely to express large DELTACUT than cows with small COWVAL and medium changes in $305 \mathrm{M}$. The medium changes in $305 \mathrm{M}$ were probably more accurate, because cows with large variation in $305 \mathrm{M}$ estimates between 2 tests were more likely to generate large DELTACUT than cows with smaller changes. The observed decrease in 305M estimates in the first month of lactation was consistent with the findings of Quist Moyer (2006), who showed that in the 
first 60 DIM, the estimates for 305M were generally overestimated.

The instability of COWVAL at the beginning of a pregnancy was expressed when, compared with cows over 60 DIM, cows whose COWVAL dropped below the baseline of $\$ 0$ in the first 60 DIM were more likely to subsequently recover to a COWVAL of $\geq \$ 500$. Interestingly, the instability in COWVAL after dropping below $\$ 0$ was observed regardless of the lactation number of the animal. These findings suggest that in the first 60 DIM, dairy managers should rely more on the test results of the previous lactation and include more than 1 COWVAL result to accurately judge the actual value of the cow within their herd. Treatments and culls early in lactation are predominantly necessary due to severe peripartal diseases (e.g., dystocia, metabolic disorders, severe clinical mastitis, or injury) and less due to production (Gröhn et al., 1998). The COWVAL module was designed as a tool to aid the farmer with a nonobvious economic culling decision. Hence, farmers are unlikely to depend solely on COWVAL in their culling decision for these early lactation cows. Overall, the farmer's perception of performance in the herd and the cow's calculated low COWVAL, predominantly early in lactation, disagreed substantially, because the farmer did not remove those cows. It needs to be emphasized that the farmer's culling decision was not based on COWVAL in this study, because COWVAL were generated retrospectively.

Based on our findings, dairy managers should limit their reliance on COWVAL for making replacement decisions on COWVAL until the number of milk tests is sufficient to accurately project the value of the cow in the current lactation. Otherwise, they might cull cows that should stay in the herd.

Disease events are not included in the calculation of COWVAL. Disease events, when they occurred and were recorded, were associated with large DELTACUT, most likely because the event may have either altered the milk production from expected or affected reproductive status. On average, cows experiencing a disease event in the $25 \mathrm{~d}$ before the COWVAL estimation had a decrease in their 305M and COWVAL, and had negative DELTA. This was especially true after 60 DIM; however, it appeared that in the first 60 DIM, it was primarily the variability in the estimated milk production, not the disease status of the cow, that led to large DELTACUT.

An abortion was often followed by a large negative DELTA (i.e., greatest median drop in COWVAL) and lowest median COWVAL. This is not surprising because abortions usually occurred later in lactation and changes in the reproductive status of cows after $60 \mathrm{DIM}$ resulted in large DELTA. On the other hand, the occur- rence of reproductive diseases (e.g., retained placenta) produced little or no effect on the calculated COWVAL or DELTA. This was probably because the majority of the reproductive diseases were recorded and treated in the voluntary waiting period (i.e., before the first AI). Consequently, in the early stage of lactation it was predominantly the change in $305 \mathrm{M}$, not the risk of a RPRODIFF (from open to pregnant), that influenced COWVAL, which could be seen in the results of the statistical model. Mastitis showed the same influence on COWVAL as lameness and metabolic and other diseases. This was probably due to a similar negative impact on the milk production of the cow. Mastitis and metabolic diseases may have led to a significant but temporary drop in the milk production or a prolonged reduction in overall milk production. Like chronic mastitis and lameness might be expected to decrease milk production to a lesser extent, but over a prolonged time. In any case, the $305 \mathrm{M}$ will be altered and hence, the COWVAL will be affected.

In this study only $2.9 \%$ of the observations had a COWVAL of $\leq \$ 0$. This reflected the high rearing cost assumed for a replacement heifer in the default values for COWVAL. With high replacement costs, there was economic pressure to retain more cows in the herd rather than replacing them. This agrees with the observations of van Arendonk (1985) and McCullough and DeLorenzo (1996), who found that low carcass prices and high costs for the replacement animal led to a reduced economic replacement rate. The study herds did not use COWVAL as a guide for culling decisions. The distribution of culling reasons for the 2 herds was consistent with reports in the literature (Bascom and Young, 1998; Smith et al., 2000; Hadley et al., 2006). It was interesting to note that culled cows had a positive COWVAL at the time of culling. This might be expected for 2 reasons. First, the positive value might reflect the high net replacement costs of $\$ 1,450$, which raised the value of the cows within the milking herd compared with the replacement heifer as mentioned previously. Second, many of the events that led to actual culling may be acute events, only recorded within $25 \mathrm{~d}$ before culling (e.g., injury, acute mastitis, calving difficulties, etc.), and therefore, did not or could not influence COWVAL. Cows that were sold or left the herd for sudden miscellaneous reasons (i.e., died or had an injury) had the greatest COWVAL at exiting. This was due to the acuteness of the disease, which did not affect the COWVAL.

As expected, cows that were removed for low production had the lowest COWVAL of the different culling categories. Low-producing cows are worth the least in a milking herd and usually show low production over a longer period, which influences COWVAL. Similar 
observations were made for the cows that were removed for reproductive failure. Due to their failure to get pregnant, they had decreased their milk production without the prospect of a future lactation. Therefore, their present and future value for the herd was minimal and COWVAL was decreased.

\section{CONCLUSIONS}

In the herds studied, after approximately 60 DIM, the Cow Value module provided a fairly stable model for the relative value of a cow in a dairy. Within the first 60 DIM, high variations in COWVAL were observed within cows and across all lactations. During this period, COWVAL may vary quite widely (by thousands of dollars) and even fluctuated above and below the baseline value of $\$ 0$ due to uncertainties in the projected 305M. Dairy producers should temper their actions during this early postpartum period by considering more information than just COWVAL when making decisions regarding an individual cow treatment, breeding, or culling. Because factors other than those included in the calculation of COWVAL may influence the suitability or a cow for culling or retention, COWVAL should be used as a guide for culling decisions, but not as the only criterion for culling.

\section{ACKNOWLEDGMENTS}

We thank Steve Eicker for his help in the generation of the dataset and general support. We are grateful for the financial support of CanWest DHI (Guelph, Ontario, Canada) and the RBB Rinderproduktion [Groß Kreutz (Havel), Germany].

\section{REFERENCES}

Bascom, S. S., and A. J. Young. 1998. A summary of the reasons why farmers cull cows. J. Dairy Sci. 81:2299-2305.

Beaudeau, F., V. Ducrocq, C. Fourichon, and H. Seegers. 1995. Effect of disease on length of productive life of French Holstein dairy cows assessed by survival analysis. J. Dairy Sci. 78:103-117.

Beaudeau, F., J. D. van der Ploeg, B. Boileau, H. Seegers, and J. P. T. M. Noordhuizen. 1996. Relationships between culling criteria in dairy herds and farmers' management styles. Prev. Vet. Med. 25:327-342.

DFO (Dairy Farmers of Ontario). 2006. Dairy Farmers of Ontario. http://www.milk.org/faqs/dairy_cattle.html Accessed Sep. 18, 2006.

Dijkhuizen, A. A., J. A. Renkema, and J. Stelwagen. 1984/85. Economic aspects of reproductive failure in dairy cattle. II. The decision to replace animals. Prev. Vet. Med. 3:265-276.

Eicker, S. W., and J. Fetrow. 2003. A prospective view of culling. http://www.wisc.edu/dysci/uwex/brochures/brochures/ EicherCulllJF. pdf Accessed Mar. 15, 2007.

Fetrow, J., K. Nordlund, and D. Norman. 2006. Culling: Nomenclature, definitions and some observations. J. Dairy Sci. 89:18961905.

Gencor. 2006. Gencor Foods Cattle Settlement Summary. http:// www.gencorfoods.ca/index.asp Accessed Sep. 18, 2006.

Groenendaal, H., D. T. Galligan, and H. A. Mulder. 2004. An economic spreadsheet model to determine optimal breeding and replacement decisions for dairy cattle. J. Dairy Sci. 87:2146-2157.

Gröhn, Y. T., S. W. Eicker, V. Ducrocq, and J. A. Hertl. 1998. Effect of diseases on the culling of Holstein dairy cows in New York state. J. Dairy Sci. 81:966-978.

Gröhn, Y. T., P. J. Rajala-Schultz, H. G. Allore, M. A. DeLorenzo, J. A. Hertl, and D. T. Galligan. 2003. Optimizing replacement of dairy cows: Modeling the effects of diseases. Prev. Vet. Med. 61:27-43.

Hadley, G. L., C. A. Wolf, and S. B. Harsh. 2006. Dairy cattle culling patterns, explanations, and implications. J. Dairy Sci. 89:2286-2296.

Kristensen, A. R. 1987. Optimal replacement and ranking of dairy cows determined by a hierarchic Markov process. Livest. Prod. Sci. 16:131-144.

Lang, B. 2003. The real cost of raising heifers. http://www.omafra.gov.on.ca/english/livestock/dairy/facts/info_co straisheif.htm Accessed Mar. 15, 2007.

Littell, R. C., G. A. Milliken, W. W. Stroup, and R. D. Wolfinger. 1996. SAS System for Mixed Models. SAS Institute Inc., Cary, NC.

Lehenbauer, T. W. 1998. Dairy cow culling decisions. Compend. Contin. Educ. Pract. Vet. 20:1362-1371.

Lehenbauer, T. W., and J. W. Oltjen. 1998. Dairy cow culling strategies: Making economical culling decisions. J. Dairy Sci. 81:264-271.

McCullough, D. A., and M. A. DeLorenzo. 1996. Effects of price and management level on optimal replacement and insemination decisions. J. Dairy Sci. 79:242-253.

Quist Moyer, M. A. 2006. Evaluation of milk production and component data from daily milk systems. MS Thesis. University of Guelph, Guelph, Ontario, Canada.

Radke, B. R., and J. W. Lloyd. 2000. Sixteen dairy culling and replacement myths. Compend. Contin. Educ. Pract. Vet. S36-S41, S56-S57.

Smith, J. W., L. O. Ely, and A. M. Chapa. 2000. Effect of region, herd size, and milk production on reasons cows leave the herd. J. Dairy Sci. 83:2980-2987.

van Arendonk, J. A. M. 1985. Studies on the replacement policies in dairy cattle. II. Optimum policy and influence of changes in production and prices. Livest. Prod. Sci. 13:101-121. 